\title{
SwissDRG, version 8.0: encourager la pertinence à l'avenir
}

\author{
Mirjam Bacha, Beatrix Meyer ${ }^{b}$ \\ ${ }^{a}$ Dr méd.,experte, $\mathrm{FMH}^{\mathrm{b}}{ }^{\mathrm{b}}$ cheffe de la division Médecine et tarifs hospitaliers, FMH
}

Le système SwissDRG devient de plus en plus difficile à saisir pour ses utilisateurs. D’une part, il n'est pas rare qu'un même thème soit remanié plusieurs fois. D’autre part, si les DRG sont homogènes du point de vue économique, souvent ils ne le sont pas du point de vue médical. SwissDRG SA a cependant mis en place des améliorations pour la représentativité des cas hautement déficitaires.

\begin{abstract}
SwissDRG SA a procédé à des changements importants dans la version 8.0. A l'instar de la version précédente, une des priorités a été d'améliorer la représentativité des cas hautement déficitaires et de réduire le nombre de cas au déficit supérieur à CHF 40000 . Le volume général du déficit des hôpitaux a baissé de $28 \%$ par rapport à la version 6.0 mais s'élève encore malgré tout à plus de CHF 120 millions. Par ailleurs, l'accent a de nouveau été mis sur la médecine intensive et les DRG de la pédiatrie.
\end{abstract}

Pour améliorer la continuité de la structure tarifaire, il serait judicieux d'examiner de plus près l'utilisation des données de plusieurs années.

Le nombre total de DRG de la version 8.0 (1037 DRG) ne diffère que très légèrement de la version précédente 7.0 (1041 DRG). Mais 13 DRG de base ont été supprimés et 40 nouveaux DRG ajoutés. Les rémunérations supplémentaires pour l'indemnisation des médicaments, procédures onéreuses, produits sanguins et implants coûteux sont passées à un nombre total de 108. Par ailleurs, certaines d'entre elles déjà existantes ont été différenciées, par exemple grâce à l'ajout de classes de dosage supplémentaires.

\section{S'atteler à un thème et le conclure au cours d'une même révision tarifaire}

Plusieurs points déjà remaniés dans la version 7.0 ont de nouveau été révisés dans la version 8.0. SwissDRG SA a modifié plusieurs fois les mêmes DRG lors de deux ou trois révisions tarifaires successives. Dans la pratique, ces changements suscitent souvent l'incompréhension, et ce notamment parce que le système de forfaits par cas devient plus complexe dans chaque nouvelle version. Cette situation complique aussi la planification des hôpitaux. Lorsqu'il est décidé de s'atteler à un thème, il serait préférable d'en achever la révision au sein d'une seule et même version. La FMH recommande d'analyser d'abord les effets des changements mis en place avant de procéder à de nouvelles modifications dans les mêmes DRG. Même si ces changements répétés sont sans doute liés à la qualité encore insuffisante des données, il serait quand même utile de les documenter et d'en indiquer la raison dans un rapport détaillé et transparent.

\section{Qualité et quantité des données: un défi récurrent}

La qualité du système SwissDRG dépend dans une large mesure de l'ensemble des données reçues. SwissDRG SA incite donc les hôpitaux à améliorer continuellement leurs données des coûts et sur les prestations. Elle intensifie les échanges avec eux et leur offre la possibilité de contrôler la plausibilité des données en cours d'année. Elle organise également des ateliers ciblés avec différentes cliniques mais, en dépit de ces efforts, l'amélioration de la qualité des données reste un défi, selon SwissDRG SA.

Indépendamment de la qualité, il est important d'avoir un ensemble de données conséquent pour la stabilité du système. Comme le montre la figure 1, plusieurs DRG font état d'une petite base de calcul pour la version en vigueur (SwissDRG 7.0). Dans ce cas, SwissDRG SA tient également compte des données de l'année précédente. ${ }^{1}$ Pour la version 8.0, SwissDRG SA a réuni les données de deux ou trois années pour le calcul de 30 DRG avec peu de cas. Pour la FMH, il serait judicieux d'examiner aussi cette manière de procéder pour les DRG avec un 


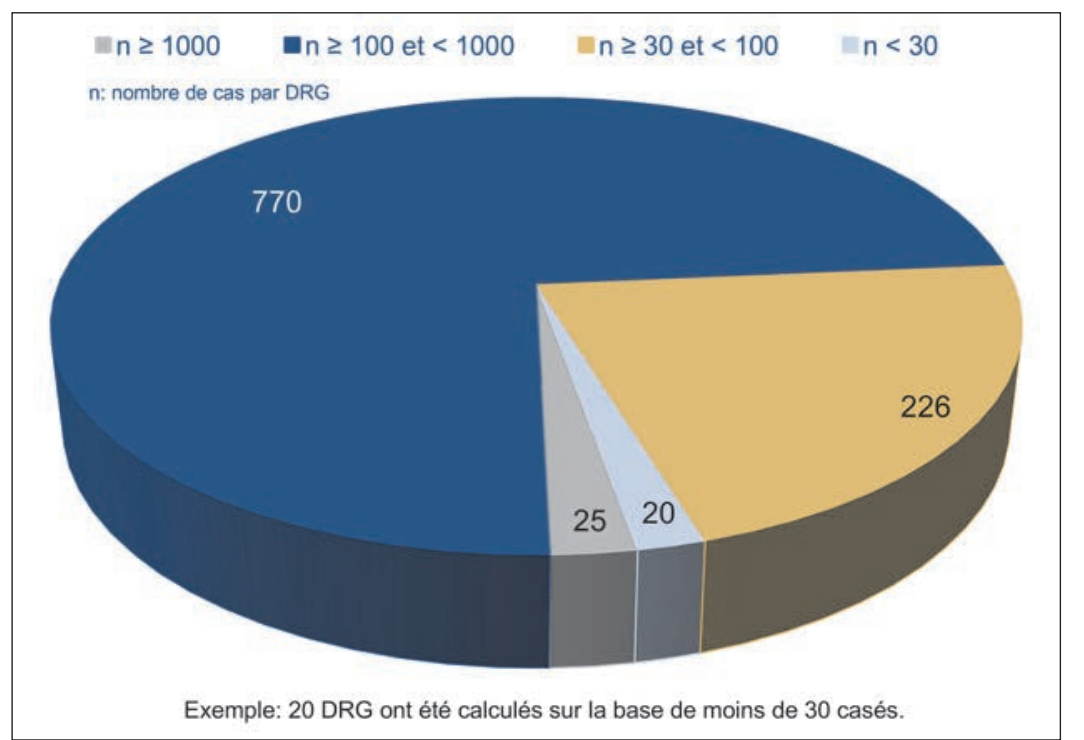

Répartition des DRG selon le nombre de cas recensés pour le calcul.

nombre important de cas. Cela permettrait de réduire les variations parfois importantes du cost-weight (CW) entre les différentes versions de SwissDRG et d'améliorer la continuité dans la structure tarifaire.

\section{Meilleure prise en compte de l'homo- généité médicale}

SwissDRG SA réunit dans un même DRG une partie des cas médicalement différents si leur nombre est faible. Il s'agit donc de vérifier si, par exemple, la compilation des données de plusieurs années pourrait permettre d'éviter ce genre de regroupement. Selon la FMH, il est important de veiller à l'homogénéité des DRG non seulement du point de vue économique mais aussi médical. Pour que le système SwissDRG soit compréhensible et puisse être perfectionné de manière cohérente, un DRG doit regrouper des prestations médicalement comparables.

Il est par ailleurs primordial d'intégrer en priorité les prestations spécifiques dans les catégories majeures de diagnostics (Major Diagnostic Category ou MDC) dédiées à un organe et précédées d'une pré-MDC réunissant des groupes de cas particulièrement chers (par ex. transplantation, ventilation de longue durée, réadaptation précoce). Une première répartition des prestations de la pré-MDC vers les MDC (spécifiques à un organe) a déjà eu lieu dans la version 7.0. Avec la version 8.0, SwissDRG SA a continué ces travaux dans divers domaines. Cette manière de procéder devrait être pour- suivie car elle permet de rendre le système SwissDRG plus clair et pertinent.

\section{Vue plus précise de la complexité des cas particuliers}

Pour représenter la complexité des tableaux cliniques, le système SwissDRG dispose du niveau de complexité clinique du patient, appelé niveau $\mathrm{PCCL}^{2}$, qui attribue une valeur entre 0 et 4 en fonction du degré de complication et/ou de comorbidité (légère, moyennement sévère, sévère ou extrêmement sévère). Malheureusement, SwissDRG SA a rejeté une demande visant à mieux différencier les cas en introduisant les niveaux 5 et 6 . Elle a justifié sa décision par une non-faisabilité technique alors que le système allemand utilise ces niveaux supplémentaires depuis des années. Tout en étant consciente de la complexité technique et opérationnelle de sa demande, la FMH souhaiterait que les exigences techniques requises pour la mise en œuvre soient réévaluées.

\section{Conclusion de la FMH}

Les nouvelles modifications accentuent encore la complexité du système SwissDRG. De ce fait, la structure tarifaire devient de moins en moins compréhensible. SwissDRG SA peut résoudre ce problème en veillant davantage à l'homogénéité médicale des DRG. Il serait par ailleurs judicieux de conclure tous les changements liés à un thème précis au sein d'une seule et même version de SwissDRG. La continuité du système pourrait

\section{Il est important de veiller à l'homogénéité} non seulement économique mais aussi médicale des DRG.

être améliorée en compilant les données de plusieurs années, même pour les DRG regroupant un grand nombre de cas. Cela faciliterait considérablement la tâche des hôpitaux pour leur planification. Enfin, la mise à disposition d'une documentation transparente serait très utile pour les utilisateurs.

\section{Crédit image}

Graphique établi par la FMH, source des données: SwissDRG SA, SwissDRG, version 7.0

Vous trouverez l'analyse détaillée de SwissDRG version 8.0 dans la prise de position de la FMH: www.fmh.ch $\rightarrow$ Tarifs hospitaliers $\rightarrow$ Positions $\rightarrow$ Prises de position. 